\title{
Casimir effect on the pull-in parameters of nanometer switches
}

\author{
W.-H. Lin, Y.-P. Zhao
}

Abstract Casimir effect on the critical pull-in gap and pull-in voltage of nanoelectromechanical switches is studied. An approximate analytical expression of the critical pull-in gap with Casimir force is presented by the perturbation theory. The corresponding pull-in parameters are computed numerically, from which one can notice the nonlinear effect of Casimir force on the pull-in parameters. The detachment length has been presented, which increases with increasing thickness of the beam.

\section{1}

\section{Introduction}

The nanoelectromechanical systems (NEMS), as an extension of microelectromechanical systems (MEMS), have turned into the hot research topic in recent years. In MEMS and NEMS, with the geometric dimension decreasing, the surface forces [1-6], replacing the body forces, take over the dominant position, which become more important for the nano-scales. In this paper, we just consider Casimir force and electrostatic force. Casimir [7] predicted an attractive force between objects. Kenneth et al. [8] have extended these considerations to real-world materials.

NEM switches are fundament building blocks for the design of NEMS applications. However, the pull-in phenomenon, an inherent instability of MEM and NEM switches, is one of annoying problems in design. By applying a voltage difference between the two electrodes, an electrostatic force is formed. At certain voltage the switches lost its stability and the gap between the switches rapidly decrease, until the two electrodes adhere. The voltage and deformation of the switches at this state are referred to as the pull-in voltage and the critical pull-in gap respectively, or shortly as the pull-in parameters of switches. Therefore, an analytical expression of the pull-in parameters could guide the design.

Received: 17 March 2003/Accepted: 18 November 2003

W.-H. Lin, Y.-P. Zhao ( $₫)$

State Key Laboratory of Nonlinear Mechanics (LNM), Institute of Mechanics, Chinese Academy of Sciences, Beijing 100080, China

e-mail: yzhao@lnm.imech.ac.cn

This work was supported by the Distinguished Young Scholar Fund of NSFC (Grant No. 10225209), key project from the Chinese Academy of Sciences (Grant No. KJCX2-SW-L2) and National '973' Project (No. G1999033103).
An analytical expression of the pull-in parameters has been given about the MEMS switches in [9]. A lumped two degrees of freedom (L2DOF) pull-in model was presented in [10]. The pull-in parameters for electrostatic torsion actuators are the pull-in voltage and pull-in angle [11, 12]. The pull-in phenomenon is widely applied in many micromachined devices that require bi-stability for their operation [13, 14]. In the above references [9-14], the Casimir and van der Waals effects are neglected. The significant effect of van der Waals force has been shown on the pull-in voltage of NEMS switches, but the effect on the critical pull-in gap has been omitted [15].

The Casimir effect in MEMS was studied in [16] and measured in [17]. In [18], a micromachined torsional device is used to determine the Casimir effect in MEMS. Casimir force has a profound influence on the oscillatory behavior of nanostructures [19].

The maximum length that will not stick to the substrate, also called detachment length is basic design parameter $[20,21]$.

The objective of the present paper is to study the effect of Casimir force on the pull-in parameters. An approximate analytical expression of the critical pull-in gap is obtained by the perturbation theory and the detachment lengths and the minimum initial gap of the cantilever and fixed-fixed beams are given, which are basic design parameters.

2

\section{Theory}

To simplify the analysis, the geometry shown in Fig. 1 is simplified to a one-dimensional (1D) lumped model as shown in Fig. 2. In the 1D lumped model, the NEMS switch is approximated by a rigid beam suspended over a ground plane using mechanical springs. Thus the only degree of freedom of the system is the gap, $r$, between the plate and the ground plane.

The equilibrium condition of the plate by Casimir, electrostatic and elastostatic forces $\left(F(r)=F_{\mathrm{C}}+F_{\text {elec }}+\right.$ $F_{\text {elas }}=0$ ) yields (see appendix 2 for expressions of the surface forces)

$\frac{\pi^{2} \hbar c w L}{240 r^{4}}+\frac{\varepsilon_{0} w L V^{2}}{2 r^{2}}-K(g-r)=0$.

The equilibrium is stable with $\partial F(r) / \partial r<0$. With voltage increasing, the gap decreases with instability condition is reached. Using the critical condition $\partial F(r) / \partial r=0$, we get

$-\frac{\pi^{2} \hbar c w L}{120 r^{4}}-\frac{\varepsilon_{0} w L V^{2}}{2 r^{2}}+\frac{K r}{2}=0$. 


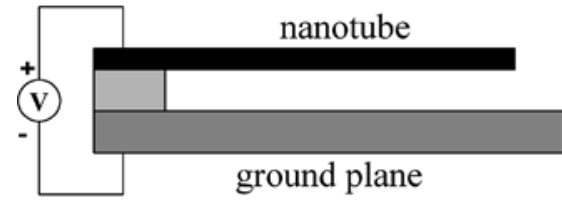

(a)

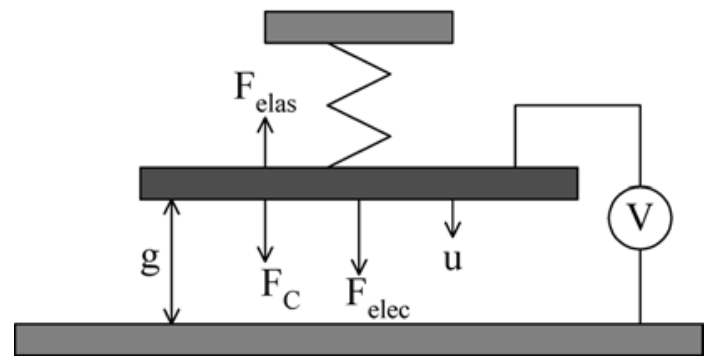

Fig. 2. One-dimensional lumped models for pull-in parameters estimation. The deflection of the plate is given by $u$, and $r=g-u$

Defining this solution by $g_{\mathrm{PI}}$ and substituting it into Eqs. (1) and (2) and then adding the equations, we obtain

$-\frac{\pi^{2} \hbar c w L}{120 g_{\mathrm{PI}}^{4}}-2 K g+3 K g_{\mathrm{PI}}=0$.

We should solve the above nonlinear equation to get $g_{\mathrm{PI}}$. by

Using Eq. (1), the pull-in voltage $V_{\mathrm{PI}}$ can be calculated

$V_{\mathrm{PI}}=\sqrt{\left[K\left(g-g_{\mathrm{PI}}\right)-\frac{\pi^{2} \hbar c w L}{240 g_{\mathrm{PI}}^{4}}\right] \frac{2 g_{\mathrm{PI}}^{2}}{\varepsilon_{0} w L}}$,

If we neglect the contribution of Casimir force in the above analysis, the critical pull-in gap and the pull-in voltage can be solved by Eqs. (3) and (4) as

$g_{0}=\frac{2}{3} g$

and

$V_{0}=\sqrt{\frac{8 K g_{0}^{3}}{27 \varepsilon_{0} w L}}$,

which are the same as the expressions derived in [9]. If we just omit the effect of Casimir force on the critical pull-in gap, that is, we replace $g_{\mathrm{PI}}$ in (4) by $g_{0}$. Then the pull-in voltage is given by

$V_{\mathrm{PI}}=\sqrt{\left[K\left(g-g_{0}\right)-\frac{\pi^{2} \hbar c w L}{240 g_{0}^{4}}\right] \frac{2 g_{0}^{2}}{\varepsilon_{0} w L}}$.

We now use the perturbation theory to discuss how much the effect of Casimir force on the critical pull-in gap around $g_{0}$. By perturbation theory, we could add a perturbation value $\delta$ around $g_{0}$, that is, we use the approximate critical value
Fig. 1. Schematic of cantilever switch: $\mathbf{a}$ cantilever switch, and $\mathbf{b}$ fixed-fixed switch

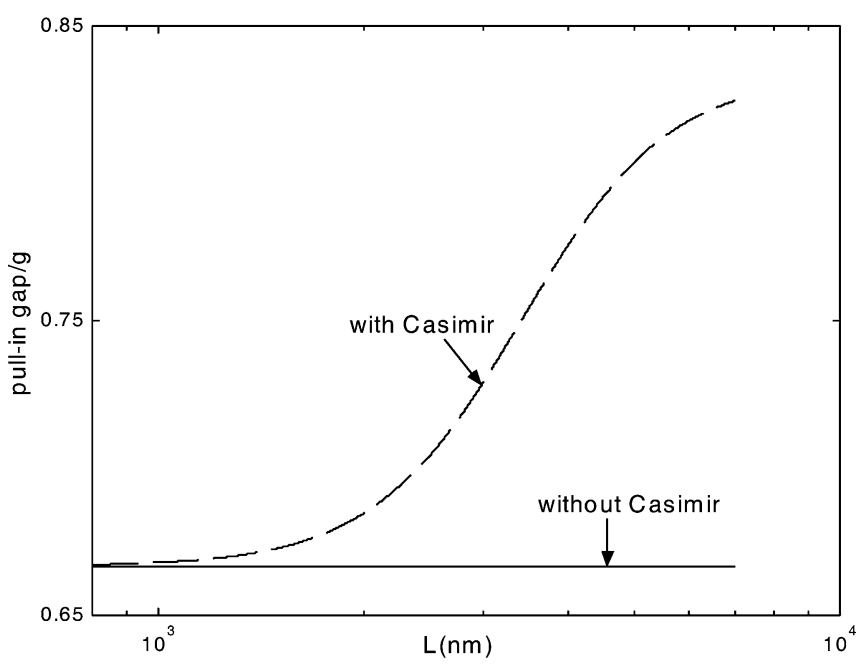

(a).

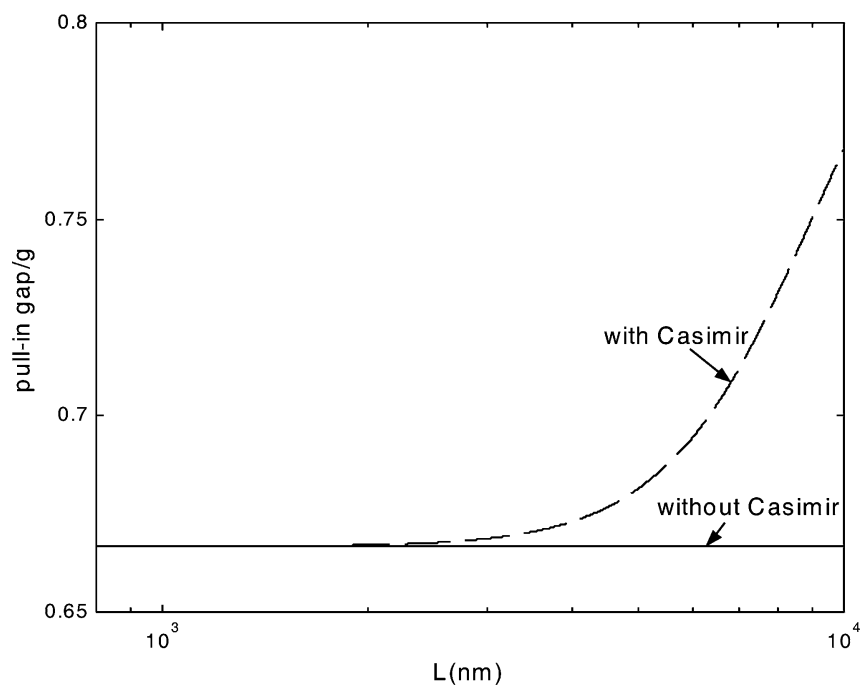

(b).

Fig. 3. Variation of the critical pull-in gap with length for the given initial gap: a Cantilever switches. b Fixed-fixed switches

$g_{\mathrm{PI}}=g_{0}+\delta$

to replace the accurate critical value.

Substituting Eq. (8) into Eq. (3), and using the firstorder approximation, i.e., neglecting the higher-order terms of $\delta$, we get the linearized function about the perturbation value $\delta$ :

$\left(K+\frac{27 \pi^{2} \hbar c w L}{320 g^{5}}\right) \delta=\frac{9 \pi^{2} \hbar c w L}{640 g^{4}}$. 


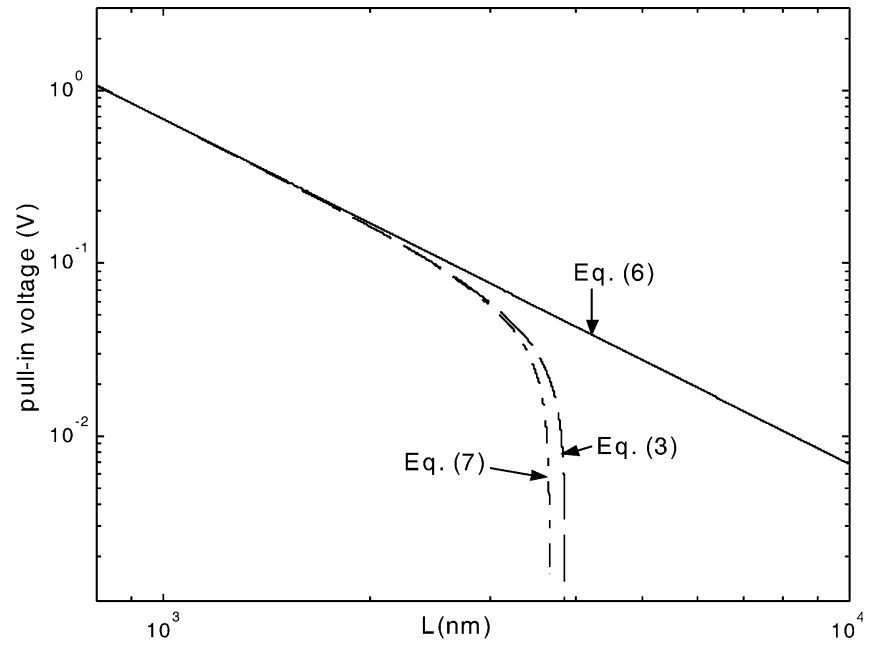

(a).

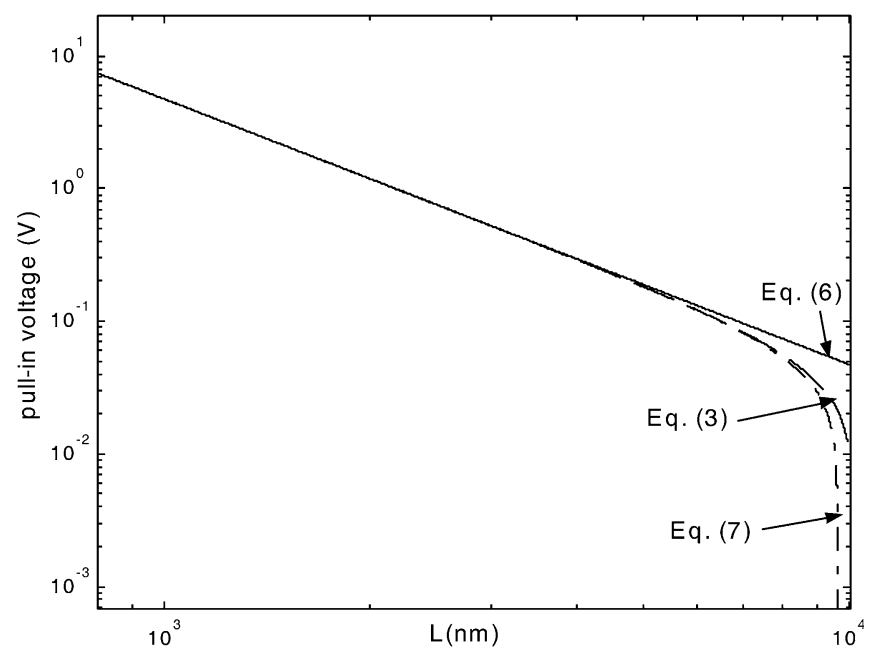

(b).

Fig. 4. Variation of the pull-in voltage with length for the given initial gap: a Cantilever switches. b Fixed-fixed switches

Solving $\delta$ from Eq. (9), we have the approximate analytical expression of the critical pull-in gap as following

$g_{\mathrm{PI}}=g_{0}+\delta$.

\section{3}

\section{Results and discussion}

Some numerical results are presented in this section. A rectangle beam is considered. Only the points with positive value for the square of the pull-in voltage are shown in Figs. 4 and 6.

\section{1}

\section{Comparison of the critical pull-in gap and the pull-in voltage}

We now consider cantilever and fixed-fixed switches with varying length and gap with the given thickness, $t=10 \mathrm{~nm}$, compared the critical pull-in parameters obtained in this paper with those results in [9].

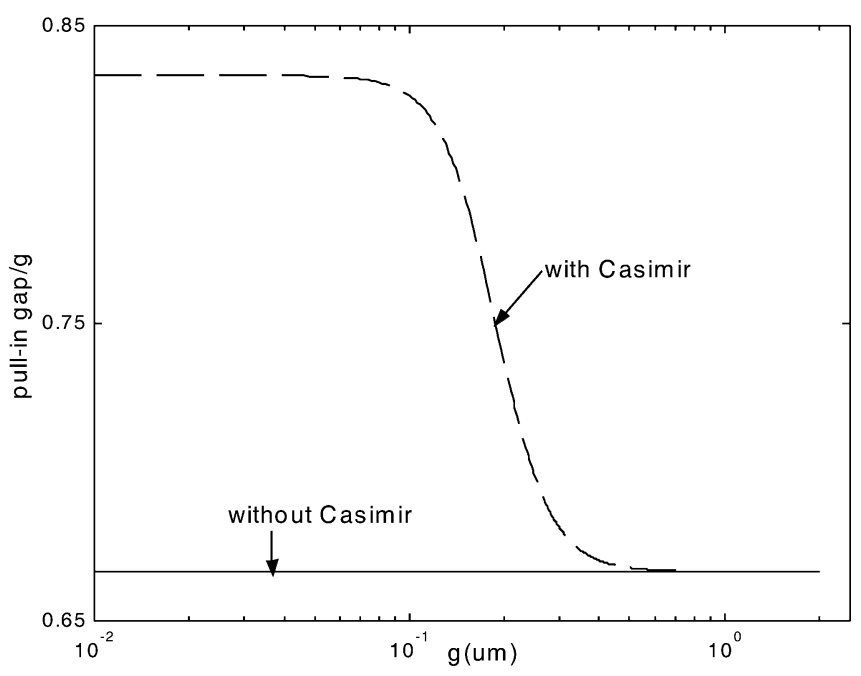

(a) Cantilever switches.



(b) Fixed-fixed switches.

Fig. 5. Variation of the critical pull-in gap with gap for the given length: a Cantilever switches. b Fixed-fixed switches

We first consider the variation of the critical pull-in gap and the pull-in voltage for the cantilever and fixed-fixed switches with length when the initial gap is $10 \mathrm{~nm}$. From Fig. 3, the critical pull-in gap with Casimir force is identical to that without Casimir force when the beam is shorter. At the same time, from Fig. 4 the pull-in voltages with Casimir force computed by (4) and (7) are also identical to that without Casimir force computed by (6). With the length increasing, the effect of Casimir force on both the critical pull-in gap and the pull-in voltage becomes much clearer. However, the difference of the pullin voltage computed by (4) and (7) is not clear. This implies that we can substitute (7) for (4) as the pull-in voltage with Casimir force for engineering designs. From Fig. 4, the square of the pull-in voltage computed by (4) or (7) is not positive when the length is larger than a critical value, the detachment length, which will be determined later. If the length is larger than the detachment length, the switch can collapse onto the ground plane even without an applied voltage. 


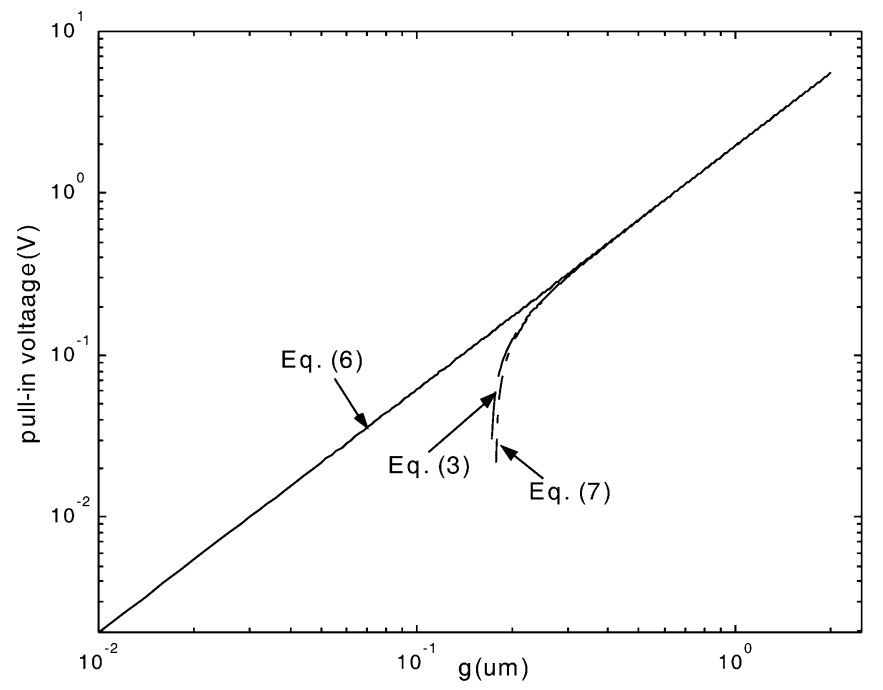

(a) Cantilever switches.

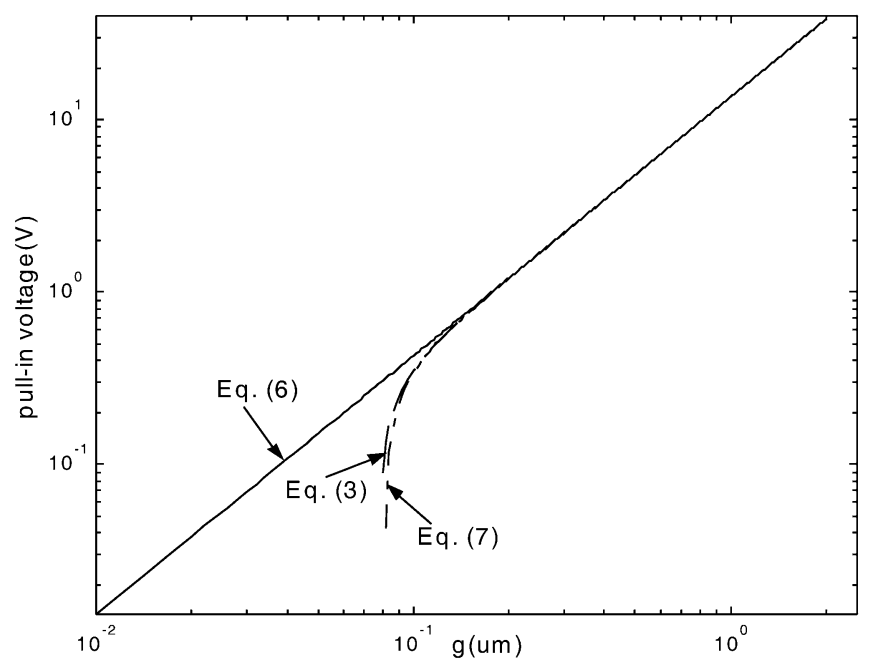

(b) Fixed-fixed switches.

Fig. 6. Variation of the pull-in voltage with gap for the given length: a Cantilever switches. b Fixed-fixed switches

Figures. 5 and 6 show the variation of the critical pullin gap and the pull-in voltage as a function of the initial gap for cantilever and fixed-fixed switches with the given length, $L=100 \mathrm{~nm}$, respectively. From Figs. 5 and 6 , the effect of Casimir on the pull-in parameters is still clear when the initial gap is less than some value, and the pullin voltage computed by (4) is still identical to that computed by (7). When the initial gap is less than a critical value, the minimum gap, the pull-in phenomenon will occur. From Figs. 3-6, we notice that the effect of Casimir force on the fixed-fixed switch is less than the effect on the cantilever switch for the same geometry parameters.

\section{2}

Comparison of the pull-in gap with different thickness

In this section, we just consider the cantilever beam. With different thickness, we compute variation of the pull-in gap with length and initial gap shown in Figs. 7 and 8,



Fig. 7. Variation of the critical pull-in gap with length with different thickness

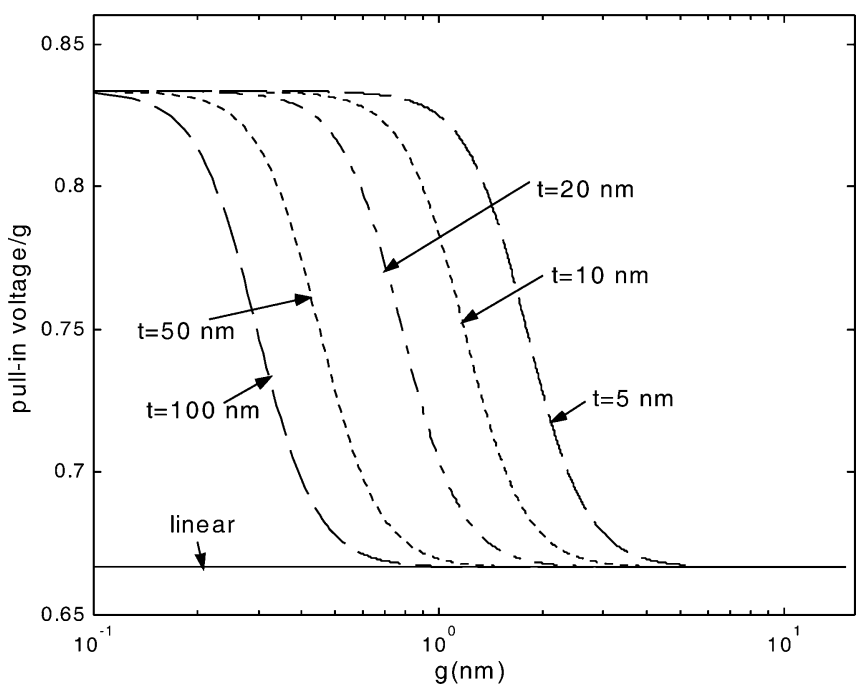

Fig. 8. Variation of the critical pull-in gap with initial gap with different thickness

respectively. From Fig. 7, we notice that the effect of Casimir force on the pull-in gap with the growth of thickness is more and more inconspicuous for the same length. That is, the detachment length increases with the increasing of the thickness of the beam. On the contrary, the minimum initial gap is decreasing with the increasing of the thickness from Fig. 8.

\section{3}

\section{Detachment parameters}

It is interesting to note that the detachment length of the cantilever and fixed-fixed beam can be obtained by equating zero the pull-in voltage in Eq. (7). That is, the detachment length of the cantilever that will not adhere with the substrate due to Casimir force is

$L_{\max }=\frac{4}{3} g \sqrt[4]{\frac{10 E t^{3} g}{3 \pi^{2} \hbar c}}$ 


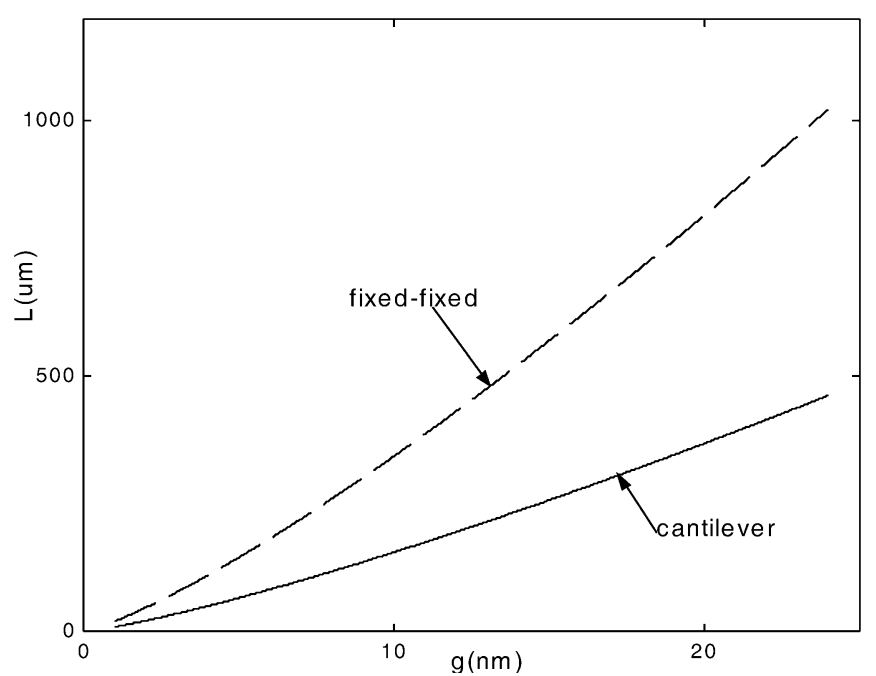

Fig. 9. Variation of the detachment length with initial gap for the cantilever and fixed-fixed switches

and the detachment length of the fixed-fixed beam is

$L_{\max }^{\prime}=\frac{4}{3} g \sqrt[4]{\frac{80 E t^{3} g}{\pi^{2} \hbar c}}$.

From Fig. 9, we could see the variation of the maximum length with gap of the cantilever and fixed-fixed switches, respectively.

As an alternative case, if the length is known, we can calculate the minimum gap. The equations of the cantilever and fixed-fixed beam are

$g_{\min }=\frac{3}{2} \sqrt[5]{\frac{\pi^{2} \hbar c L^{4}}{80 E t^{3}}}$

and

$g_{\min }^{\prime}=\frac{1}{4} \sqrt[5]{\frac{81 \pi^{2} \hbar c L^{4}}{20 E t^{3}}}$,

respectively. From Fig. 10, we could see the variation of the minimum gap with length of the cantilever and fixedfixed switches, respectively.

\section{4}

\section{Conclusion}

An approximate analytical expression of the critical pull-in gap considered Casimir force is given by the perturbation theory in this paper. Numerical results with cantilever and fixed-fixed switches show: The critical pull-in gap and the pull-in voltage with Casimir force is much more different from that without Casimir force as the geometry parameters are greater or less than some value. When the length is greater than the detachment length or the initial gap is less than the minimum gap, the beams will adhere to the substrate without any applied voltage. However, we can still substitute the critical pull-in gap, $g_{0}$ (without Casimir force), for the critical pull-in gap, $g_{\text {PI }}$ (with Casimir force), to compute the pull-in voltage with Casimir force. This would be much more convenient for engineering designs.

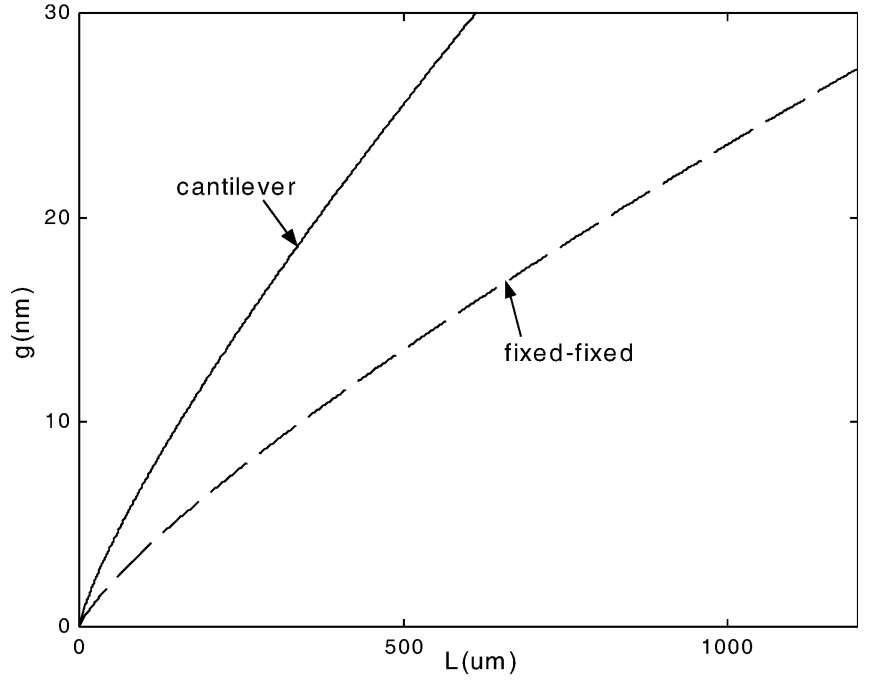

Fig. 10. Variation of the minimum gap with length for the cantilever and fixed-fixed switches

The detachment lengths of the cantilever and fixed-fixed beam have been determined by equating zero the pull-in voltage, which are fundamental design parameters for NEM switches. The detachment length is increasing or the minimum initial gap is decreasing with the growth of the cantilever beam's thickness from Figs. 7 and 8.

\section{Appendix 1}

Table 1. Parameters in the present paper

\begin{tabular}{lll}
\hline Symbol & Physical meaning & Dimension \\
\hline$c$ & Speed of light & $\mathrm{LT}^{-1}$ \\
$E$ & Effective modulus of beam & $\mathrm{ML}^{-1} \mathrm{~T}^{-2}$ \\
$g$ & Initial gap between movable & $\mathrm{L}$ \\
$\hbar$ & $\quad$ and ground plates & \\
$I$ & Planck's constant divided by $2 \pi$ & $\mathrm{ML}^{2} \mathrm{~T}^{-1}$ \\
$I$ & Moment of the inertia of & $\mathrm{L}^{4}$ \\
$K$ & $\quad$ cross-section & \\
$L$ & Effective spring constant of beam & $\mathrm{MT}^{-2}$ \\
$r$ & Length of beam & $\mathrm{L}$ \\
& Gap between movable and & $\mathrm{L}$ \\
$t$ & ground plates & $\mathrm{L}$ \\
$w$ & Thickness of beam & $\mathrm{L}$ \\
$V$ & Width of beam & $\mathrm{ML}^{2} \mathrm{~T}^{-2} \mathrm{Q}^{-1}$ \\
$\varepsilon_{0}$ & Voltage applied & $\mathrm{M}^{-1} \mathrm{~L}^{-3} \mathrm{~T}^{2} \mathrm{Q}^{2}$ \\
\hline
\end{tabular}

\section{Appendix 2}

The Casimir force between parallel plates of infinite conductivity separated be a distance $r$ is given by [7]

$\frac{F_{\mathrm{C}}}{w L}=-\frac{\delta U}{\delta r}=\frac{\pi^{2} \hbar c}{240 r^{4}}$,

where $w$ and $L$ are the width and length of beam, respectively, $\hbar=1.055 \times 10^{-34}$ Js is the Planck's constant divided by $2 \pi, c=2.998 \times 10^{8} \mathrm{~m} / \mathrm{s}$ is the speed of light. 
For the parallel plate configuration shown in Fig. 2, the electrostatic force (not accounting for fringing fields) is given by [9]

$F_{\text {elec }}=\frac{\varepsilon_{0} w L V^{2}}{2 r^{2}}$,

where $V$ is the applied voltage, $\varepsilon_{0}=8.854 \times 10^{-12} \mathrm{C}^{2} \mathrm{~N}^{-1} \mathrm{~m}^{-2}$ is the permittivity of vacuum within the gap.

The elastostatic force, $F_{\text {elas }}$, is modeled by a spring. The effective spring constant, $K$, is derived from the small-deflection mechanical solution for the maximum displacement, $g_{\max }$, of the structure with a uniform distributed load. Considering an 1D lumped beam model, the spring constant is $K=8 E I / L^{3}$ for a cantilever beam and $K=384 E I / L^{3}$ for a fixed-fixed beam, where $E=1.2 \mathrm{TPa}$ is the Young's modulus, and $I$ is the moment of inertia.

\section{References}

1. Israelachvil JN (1985) Intermolecular and surface forces, Academic Press

2. Maboudian R; Howe RT (1997) Critical review: adhesion in surface micromechanical structures. J Vac Sci Technol B 15(1): 1-19

3. Komvopoulos K (1996) Surface engineering and microtribology for microelectromechanical systems. Wear 200: 2305-2327

4. Zhao YP (2002) Morphological stability of epitaxial thin elastic films by van der Waals force. Arch Appl Mech 72(1): 77-84

5. Zhao YP; Li WJ (2002) Surface stability of epitaxial elastic films by the Casimir force. Chin Phys Lett 19(8): 1161-1163

6. Zhao YP; Wang LS; Yu TX (2003) Mechanics of adhesion in MEMS-a review. J Adhesion Sci Technol 17: 519-546

7. Casimir HBG (1948) Proc K Ned Akad Wet 51: 793.

8. Kenneth $\mathrm{O}$ et al (2002) Repulsive Casimir force. Phys Rev Lett 89: 033001
9. Osterberg PM (1995) Electrostatically actuated micromechanical test structures for material property measurement. PhD Dissertation, MIT, Cambridge, MA

10. Bochobza-Degani O; Nemirovsky Y (2002) Modeling the pullin parameters of electrostatic actuators with a novel lumped two degrees of freedom pull-in model. Sensors \& Actuators A 97-98: 569-578

11. Degani O; Nemirovsky Y (2002) Design considerations of rectangular electrostatic torsion actuators based on new analytical pull-in expressions. J Microelectromech Syst 11(1): 20-26

12. Degani O; Socher E; Lipson A; Leitner T; Setter DJ; Kaldor S; Nemirovsky Y (1998) Pull-in study of an electrostatic torsion microactuator. J Microelectromech Syst 7(4): 373-379

13. Zhang LX; Zhao YP (2003) Electromechanical model of RF MEMS switches. Microsystem Technologies 9: 420-426

14. Hornbeck LJ (1991) Spatial light modulator and method, US Patent 5,061,049

15. Dequesnes M; Rotkin SV; Aluru NR (2002) Calculation of pull-in voltages for carbon-nanotube-based nanoelectromechanical switches. Nanotechnology 13: 120-131

16. Serry FM; Walliser D; Maclay GJ (1998) The role of the Casimir effect in the static deflectionand stiction of membrane strips in MEMS. J Appl Phys 84(50): 2501-2506

17. Chan HB; Aksyuk VA; Kleiman RN; Bishop DJ; Capasso F (2001) Quantum mechanical actuation of microelectromechanical systems by the Casimir force. Science 291: 1941-1944

18. Chan HB; Aksyuk VA; Kleiman RN; Bishop DJ; Capasso F (2001) Nonlinear micromechanical Casimir oscillator. Phys Review Lett 87(21): 211801

19. Bukes E; Roukes ML (2001) Stiction, adhesion energy, and the Casimir effect in micromechanical systems. Phys Rev B 63: 033402

20. Zhao YP (2003) Stiction and anti-stiction in MEMS and NEMS. Acta Mechanica Sinica 19(1): 1-10

21. Johnstone RW; Parameswaran M (2002) Theoretical limits on the freestanding length of cantilever produced by surface micromachining technology. J Micromech Microeng 12: 855-861 